ISBN 978-981-14-1684-2

Proceedings of 2019 the 9th International Workshop on Computer Science and Engineering

(WCSE 2019 SUMMER)

Hong Kong, 15-17June, 2019, pp. 863-868

doi: $10.18178 /$ wcse.2019.06.129

\title{
The design of Push-down magnetic levitation system
}

\author{
Wang Yongliang ${ }^{1}$, Li Xinqi $^{1}$, Chen Hongdou ${ }^{2+}$, Yan Daliang ${ }^{1}$, Lu Dengcheng ${ }^{1}$ \\ 1 School of Automation and Electrical Engineering, Tianjin University of Technology and Education, \\ Tianjin ${ }^{2}$ Sanshui District Industrial Secondary School in Foshan City, Guangdong Province, China
}

\begin{abstract}
Magnetic levitation technology has the characteristics of no friction, no pollution, low noise, etc., so it has very important value in aerospace and mechanical manufacturing. In recent years, with the development of new high magnetic materials and electromagnetic theory. At present, it has become a hot research field of major universities and research institutes. At the same time, the magnetic levitation system is a complex and unstable system with mufti-degree of freedom, strong coupling and nonlinear fastness. Therefore, the magnetic levitation control system has become a hot spot and a difficult point in the field of control science. In this paper, a hardware platform of push-down magnetic levitation control system is constructed. The magnetic field of the floating magnetic field on the vertical component is collected by the Honeywell 3503 sensor placed diagonally. The linear signal is converted into an electrical signal and amplified by the operational amplifier. The STM32 reads and writes control information and uses it for the digital filtering algorithm and the run control algorithm to apply the calculated data to the power amplifier component to drive the helical coil to generate a corrected magnetic field. Keep the float in dynamic balance and achieve the desired control effect.
\end{abstract}

Keywords: magnetic levitation control; anti-integration saturation PID; STM32

\section{Introduction}

Magnetic levitation technology is referred to as technology, or technology. In principle, it mainly utilizes the magnetic field characteristics in physics to cause objects to float in the air. At present, we mainly apply it to urban rail transit. Although large-scale use has only begun in modern times, in reality, magnetic levitation is a long-established technology. According to the theory, we can find that magnetic levitation technology was proposed by a German engineer in the early $1920 \mathrm{~s}^{[1]}$. Rifleman Keeper, not only that, magnetic levitation technology has been widely concerned by the scientific community since its inception ${ }^{[2-3]}$. After more than ten years of existence, magnetic levitation technology has successfully applied for a patent. It can be said that magnetic levitation technology is An important scientific discovery in the history of human society has theoretically promoted the continuous development of driving engineering.

In general, magnetic levitation technology has a complete discipline theory. With the continuous development of China's scientific and technological capabilities and the increasing demand of human beings, magnetic levitation technology has emerged as a practical subject, and has promoted mutual cooperation with power electronics technology, control theory and control engineering, and other technologies. The goal of development. Among them, research on magnetic levitation transport vehicles, metal smelting and magnetic suspension bearings has become a hot point at home and abroad, and Germany and Japan are leading in magnetic levitation transport vehicles.

This paper designs the hardware platform of the push-down magnetic levitation system based on STM32 design, and improves the control algorithm to achieve the floating point fixed-point anti-disturbance suspension.

+ Corresponding author. Tel.: +13622133013

E-mail address: china68kun@163.com. 


\section{System Principle}

The entire control system of the push-down magnetic levitation includes a power module, a position measuring module, a power amplifying module and a main controller module. Its structure is shown in Figure 1. The entire power supply of the chip comes from the power module. This paper designs three inboard power supplies, $12 \mathrm{~V}, 5 \mathrm{~V}$, and $3.3 \mathrm{~V}$. The $3.3 \mathrm{~V}$ voltage is supplied to the $\mathrm{STM} 32$ main controller, the $5 \mathrm{~V}$ voltage is supplied to the position detection module chip and the driver chip is used, and the $12 \mathrm{~V}$ voltage is supplied to the drive module. The position measuring module dynamically measures the spatial position information of the float. Compared with the target position, it is an important part of achieving closed-loop control. The data collected by the position measurement module and the data recorded during the debugging process are processed by the main control chip, and the driving power information is obtained in the form of PWM control wave by the PID algorithm.

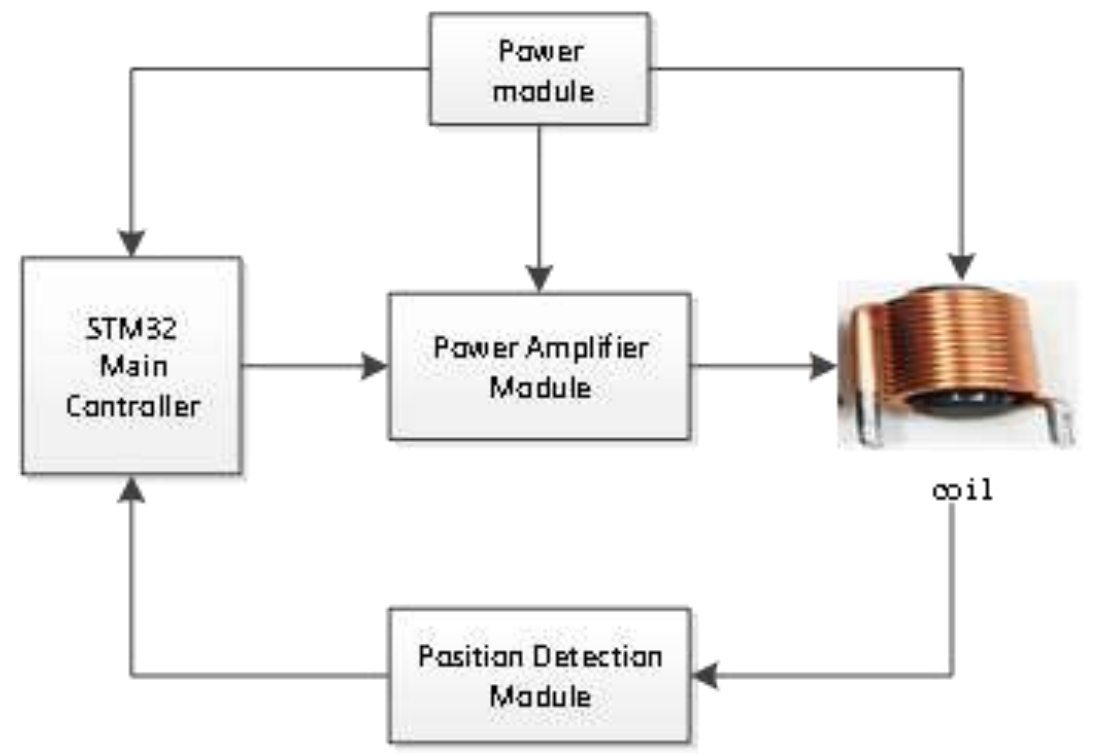

Fig. 1: System schematic block diagram

\section{Design of Hardware System}

\subsection{Power module}

The power module is the energy source for the operation of the electronic circuit and is an indispensable part of the electronic circuit. Moreover, the quality of the power supply is related to the stability of the operation of the chip and its circuit. Therefore, we should make the power module fully meet the power and ripple factor required by the external circuit, and try to improve the efficiency of the power supply.

In a magnetic levitation system, the control circuit has the following requirements for the power supply:(1) Since the coil and its power amplifying component have low requirements on the stability of the power supply, but the power requirement is high, the power supply of the coil and its power amplifying component is directly powered by the $12 \mathrm{~V}$ lithium battery power supply.(2) The power requirements of the main controller module and the position measurement module are not high, but the stability of the voltage is very high. Therefore, it is necessary to provide a voltage regulator to regulate the power supply. This article is designed to use a regulated voltage of $3.3 \mathrm{~V}$, and a $5 \mathrm{~V}$ step-down regulated power supply. The AMSII7 power chip is used as a 3.3V step-down regulator chip (Figure 2). The Lm2596S power chip is used as a $5 \mathrm{~V}$ step-down regulator chip. A small amount of electronic components can be added to the periphery of the chip to complete the voltage amplitude adjustment. The frequency is internally provided with a frequency generating device and a frequency compensating device. In a similar switching frequency device, no complicated filtering components are required. Its circuit schematic is shown in Figure 3. 


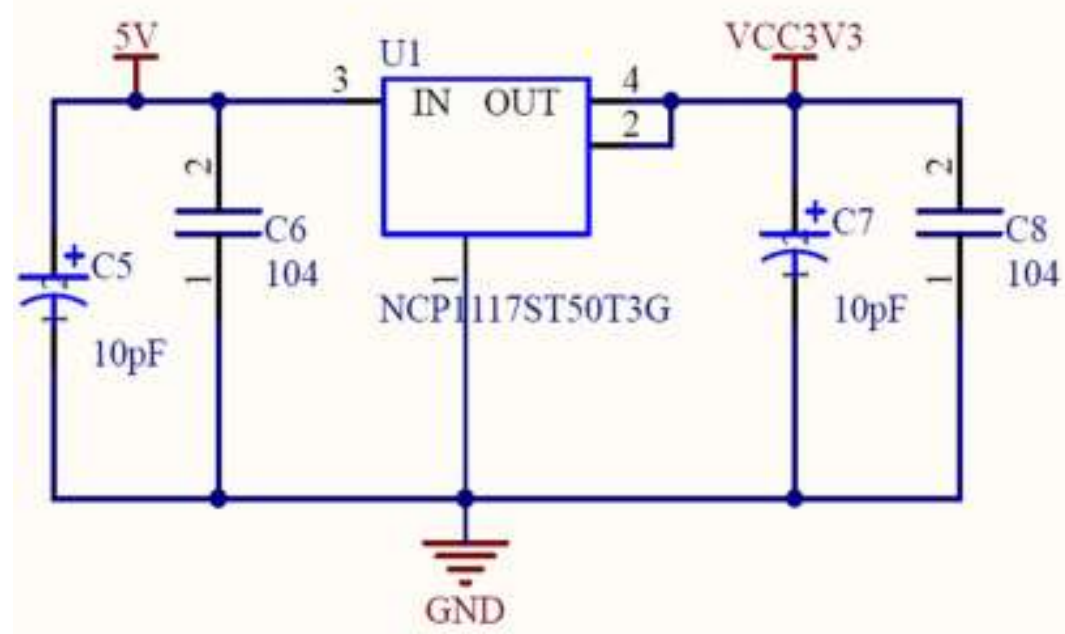

Fig. 2: Voltage stabilizing circuit

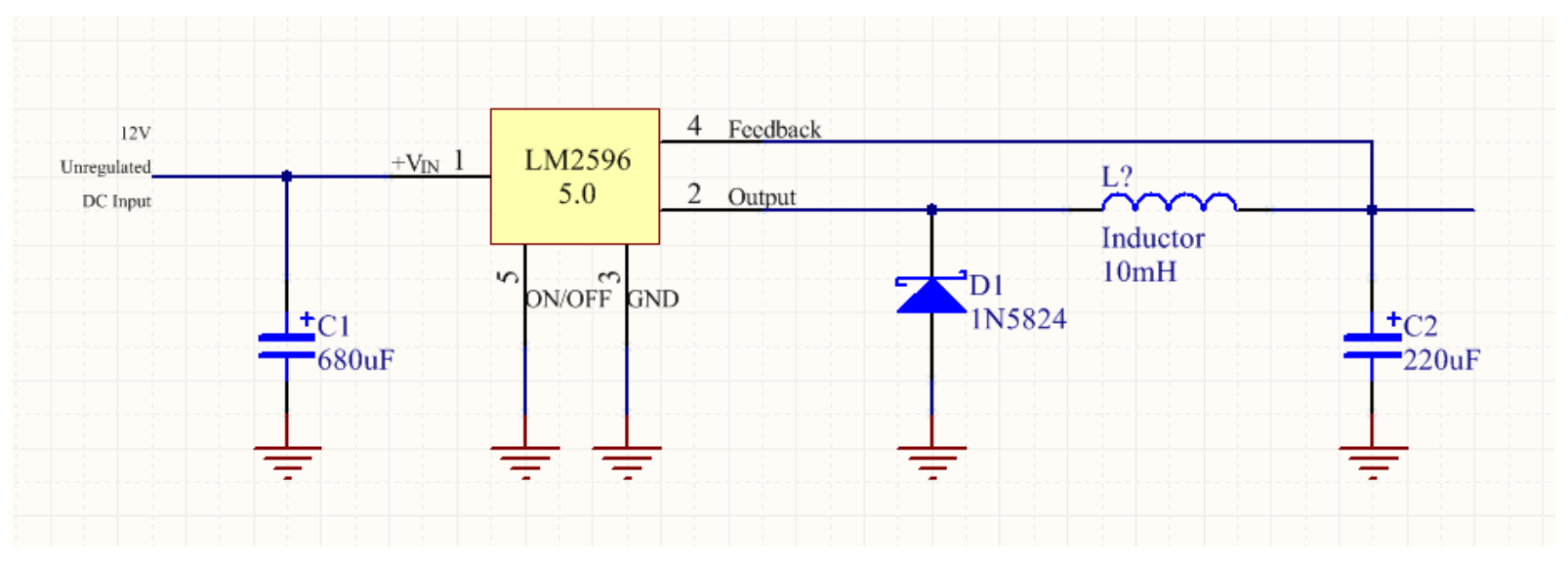

Fig. 3: Voltage stabilizing circuit

\subsection{Position measurement module}

The position detection module is an external factor that improves the control accuracy of the magnetic levitation system. It is the key link to achieve control. The measurement of position in dynamic space plays an increasingly important role in modern production as an important measure of industrial production. This system uses the HAL3503 series linear Hall circuit as the position measurement module.

The position measurement module consists of a 3503 Hall voltage generator and a LM324 linear amplifier. The input is the magnetic induction intensity of the float magnet on the vertical plane. After the 3503 Hall voltage generator, it will be converted into a voltage signal. At this time, the voltage signal will change linearly with the magnetic induction intensity, but the internal contains weak interference signals. And the voltage variable range is relatively small. If this signal is directly input into the controller, the acquisition value of the ADC changes relatively small, and the position information acquired at this time is inaccurate due to the interference signal, even if the selection of the control algorithm is appropriate, the height of the float magnet will be high. Frequency oscillation. It is not even possible to reach equilibrium. 


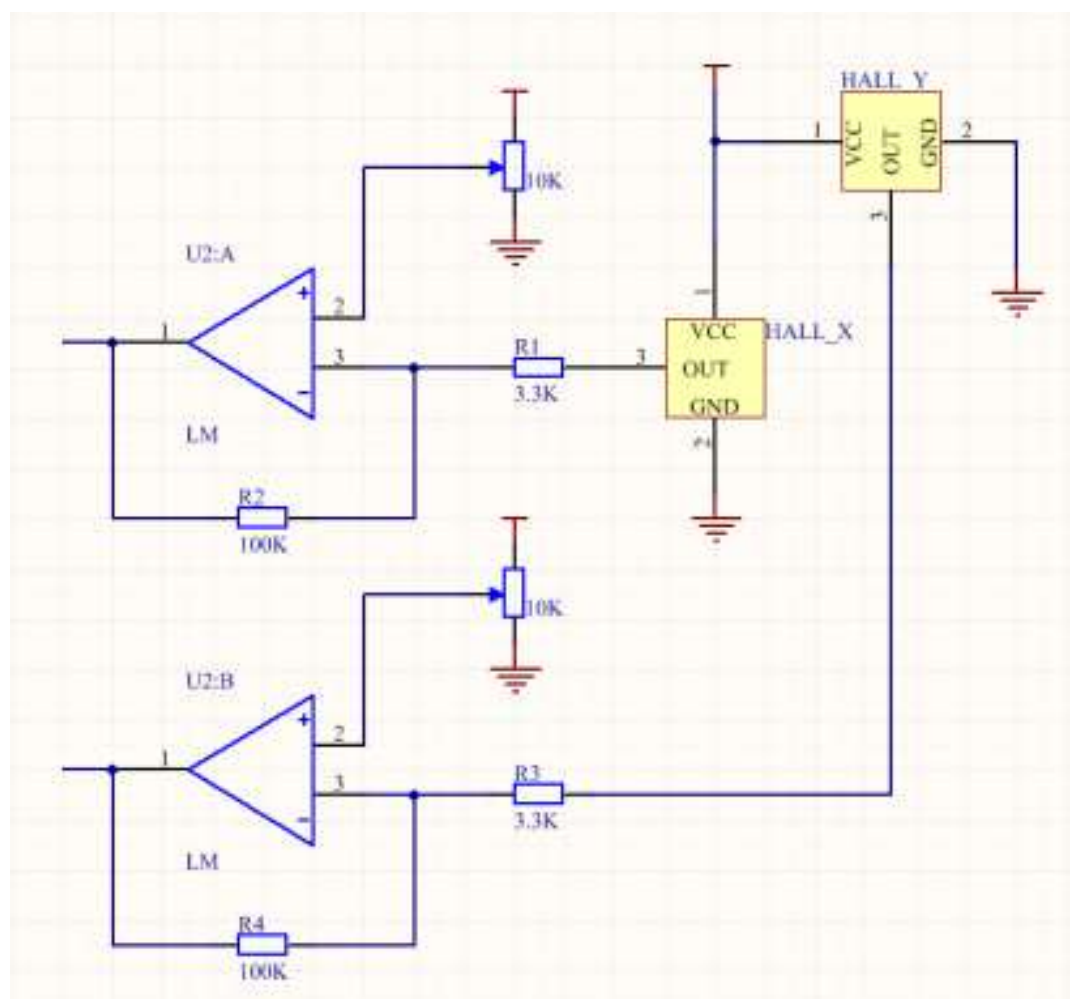

Fig. 4: Circuit schematic diagram of position measurement module

To avoid this, we added the LM324 linear amplifier and follower to the back of the 3503 Hall voltage generator. Adjusting the variable resistor makes the float at the intermediate equilibrium position voltage $2.5 \mathrm{~V}$. At this time, the LM324 is in the linear amplification region, and then the corresponding low-pass digital filtering algorithm is combined to obtain an applicable position signal.

\section{System Software Design}

The push-down magnetic levitation control system includes system hardware and system software. The hardware system is the basis for achieving the control objectives. The realization of each unit function of the magnetic levitation system is based on the hardware system. The software part of the magnetic levitation control system is the core of the realization of specific functions. It controls the hardware structure of the system and the mechanical structure of the external mechanical structure to be closely linked to achieve specific functions. During the balance adjustment process, the direction of motion, acceleration and response to external disturbances are all achieved by the control system software through the corresponding control algorithm correction. Therefore, designing the control system software and the algorithms and parameters it requires are critical to the overall magnetic levitation system.

The software design mainly includes two parts: The first part is the design of STM32F103 processor module software. The main task is to debug the position detection module ADC acquisition, drive module PWM control, etc., and initially complete the closed-loop control network according to the framework of the PID algorithm. The second part is based on the actual object to debug and improve the algorithm. The second part is described during the commissioning process.

After the magnetic levitation system is powered on, the system initializes the register status of each aspect and the controller parameters. System initialization mainly includes: system initial state configuration initialization, GPIO initialization, global variable initialization, initial state establishment, and so on. GPIO initialization includes: USART1 serial communication port initialization, drive module PWM signal port initialization configuration, timer 2 initialization configuration, and ADC DMA initialization configuration. 
Global variable initialization includes: initialization of ADC acquisition information, initialization of status flag bits, initialization of serial port receive buffer, initialization of PID parameters, and so on.After the control system is initialized, the entire system is ready for servo control, and each sub-module has started to work normally. As shown in Figure 5, it is the main function program flow chart of the magnetic levitation system.

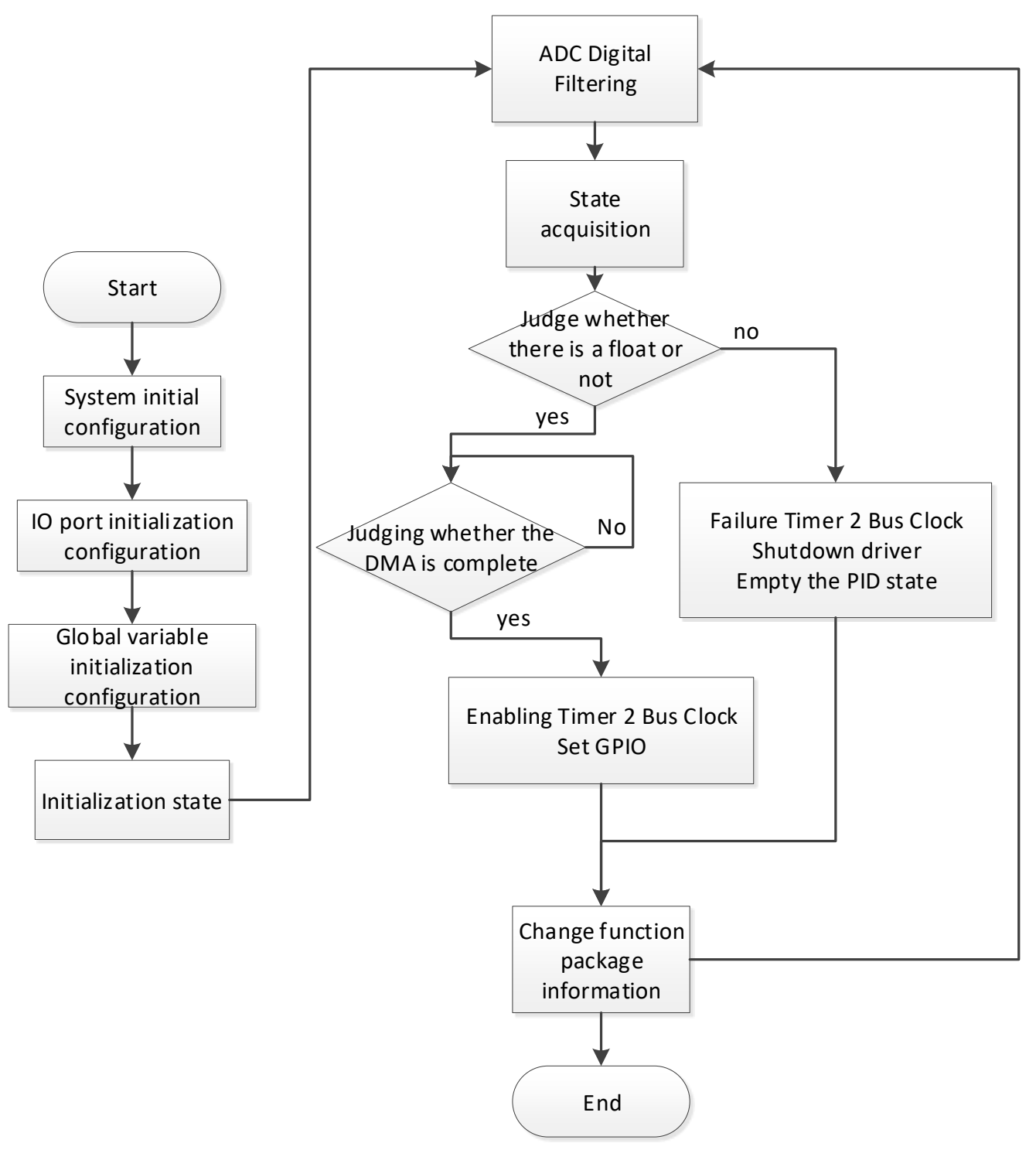

Fig. 5: The main function program flow chart of the magnetic levitation system

During the operation of the system, it is necessary to judge whether the float is placed in the controllable periphery, that is, whether the float is placed. If it is no longer within the control range, it is necessary to turn off the drive, clear the PID data packet, place the coil overheated, or have a large error when placed next time. accumulation. Change the function package entry parameters to implement the call of different function functions.

\subsection{Debugging and improvement of PID algorithms}

When debugging the PID, first set P to 1 , and set the two parameters of I D to 0 , increase P, so that the floating system can respond quickly when it exceeds the set position, so that it has obvious recovery force. At this point, the float is not balanced, and it can feel a large shock, and the shock will continue to expand. The frequency of the shock is not large.

After that, consider increasing the parameter D to reduce the low frequency oscillations. When adjusting to low frequency oscillations to eliminate high frequency oscillations. Change the PD parameters in the same 
proportion, adjust the float to suspend, and will not fall. However, it will be found that the float does not stabilize in the center position, but rather moves irregularly around the center position.

This is due to static errors. In order to eliminate static errors, we can add I parameters in a small amount. Due to the limitation of integral amplitude, the integral action does not make the system unbalanced, and it can eliminate certain static errors when the distance is close.Since the PD function is much stronger than the effect of I, it is found that when the float has large interference, the error cannot be eliminated. And always exist.

During the debugging process, it was found that the balance can be achieved after debugging the float by the PD algorithm, but the float is randomly oscillated in an area with a small distance from the equilibrium position. After adding the I parameter, the float can not only eliminate the static error but also lose the float. Balanced, after debugging analysis, it is found that the integral operation in PID can theoretically eliminate the static deviation of the system, but it also has a fatal deficiency for this kind of strong lag system, which will cause integral saturation. That is to say, when the deviation always exists, it will lead to too much integration. When the direction of the float changes, it takes a period of adjustment to eliminate the effect of the original integral. This causes adjustment lag. Let the system return to an unbalanced state. Consider the post-trial integral limit to improve the original PID computer system. When the integral term output reaches the output integral upper limit, the calculation of the integral term is stopped. This eliminates static errors in a small range from the equilibrium position without causing an imbalance in the system due to integral saturation. The block diagram of the integral limiter PID .

\section{Results and Analysis}

This paper constructs the hardware platform of the push-type magnetic levitation system, and designs the supporting software to control the system. Through the adjustment and improvement of the control algorithm, the floating point fixed-point anti-disturbance suspension is finally realized. The hardware system physical map is shown in Figure 6.

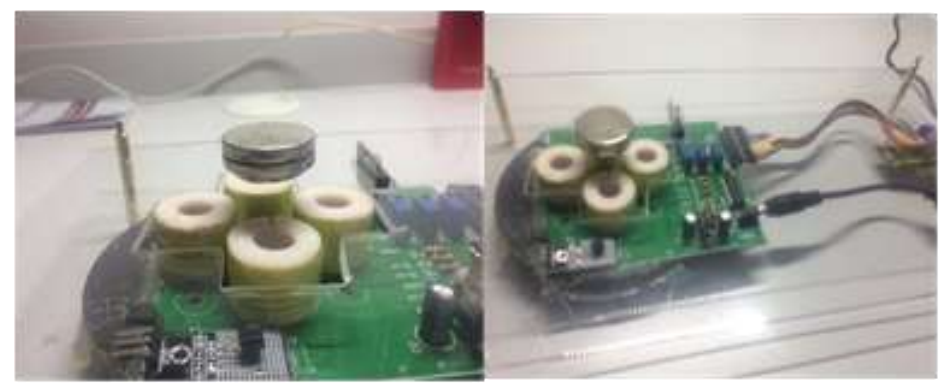

Fig. 6: The actual physical test diagram of the system

\section{Acknowledgments}

This work was supported by Innovation and Entrepreneurship Training Projects for Tianjin College Students (Project No.201810066019), the Foundation of Tianjin University of Technology and Education (Project No.KJ15-06), and the Foundation of Tianjin University of Technology and Education(Project No.YC19-17)

\section{References}

[1] Y.Z.Tian,S.J.Sun,H.R.Lin,Z.F.Zhang.Fatigue behavior of $\mathrm{CoCrFeMnNi} \mathrm{high-entropy} \mathrm{alloy} \mathrm{under} \mathrm{fully} \mathrm{reversed}$ cyclic deformation[J].Journal of Materials Science \&amp; Technology,2019,35(03):334-340.

[2] Ye Yuan,Yukun Sun,Qianwen Xiang.Design and Analysis of a Magnetic Bearings with Three Degrees of Freedom[J].Chinese Journal of Mechanical Engineering,2019,32(01):142-151.

[3] Kong Deshan. Research on subway acceleration measurement method based on magnetic levitation technology[A]. IEEE Beijing Section、Chinese Instrument and Control Society (CIS).Proceedings of 2017 13th IEEE International Conference on Electronic Measurement \&amp; Instruments (ICEMI)[C].IEEE Beijing Section、 Chinese Instrument and Control Society (CIS):IEEE BEIJING SECTION,2017:7. 